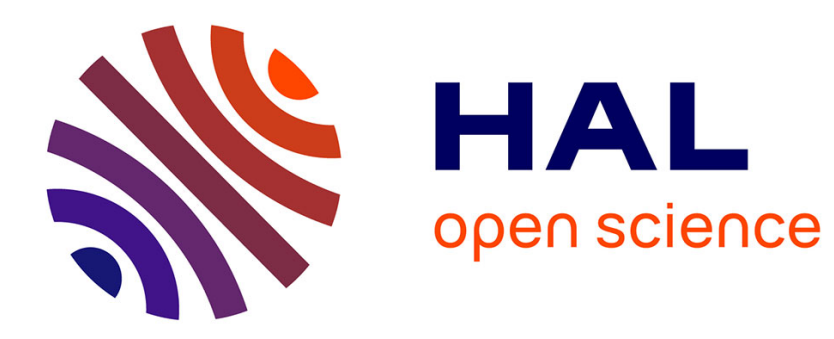

\title{
On enveloping type-definable structures
}

Cédric Milliet

\section{To cite this version:}

Cédric Milliet. On enveloping type-definable structures. The Journal of Symbolic Logic, 2011, 76 (3), pp.1023-1034. 10.2178/jsl/1309952532 . hal-00582389

\section{HAL Id: hal-00582389 \\ https://hal.science/hal-00582389}

Submitted on 1 Apr 2011

HAL is a multi-disciplinary open access archive for the deposit and dissemination of scientific research documents, whether they are published or not. The documents may come from teaching and research institutions in France or abroad, or from public or private research centers.
L'archive ouverte pluridisciplinaire HAL, est destinée au dépôt et à la diffusion de documents scientifiques de niveau recherche, publiés ou non, émanant des établissements d'enseignement et de recherche français ou étrangers, des laboratoires publics ou privés. 


\title{
ON ENVELOPING TYPE-DEFINABLE STRUCTURES
}

\author{
CÉDRIC MILLIET
}

\begin{abstract}
Aвstract. We observe simple links between preorders, semi-groups, rings and categories (and between equivalence relations, groups, fields and groupoids), which are type-definable in an arbitrary structure, and apply these observations to small structures. Recall that a structure is small if it has countably many pure $n$-types for each integer $n$. A $\emptyset$-type-definable group of finite arity in a small structure is the conjunction of definable groups. A $\emptyset$-type-definable category of finite arity in a small structure is the conjunction of definable categories. For an $A$-type-definable group $G_{A}$ of finite arity (where the set $A$ maybe be infinite) in a small and simple structure, we deduce that

1) if $G_{A}$ is included in some definable set $X$ such that boundedly many translates of $G_{A}$ cover $X$, then $G_{A}$ is the conjunction of definable groups.

2) for any finite tuple $\bar{g}$ in $G_{A}$, there is a definable group containing $\bar{g}$.
\end{abstract}

In a universe $\mathfrak{M}$, a $A$-type-definable set, instead of being defined by a formula, is the conjunction of infinitely many formulae with parameters in some set $A$. A A-typedefinable structure in $\mathfrak{M}$ is any structure whose domain, functions and relations are $A$-type-definable in $\mathfrak{M}$.

Definition. Let $\mathcal{S}$ be a class of structures, and $A$ an element of $\mathcal{S}$ which is typedefinable in $\mathfrak{M}$. We say that $\mathfrak{M}$ loosely envelopes $A$ with respect to $\mathcal{S}$ if $A$ is contained in some definable structure belonging to $\mathcal{S}$. We say that $\mathfrak{M}$ envelopes $A$ with respect to $\mathcal{S}$ if $A$ is the conjunction of definable structures in $\mathcal{S}$.

In the sequel, the class $\mathcal{S}$ will consist either of groups, semi-groups, fields, rings, preorders, equivalence relations, categories or groupoids and will be obvious from the context. For instance, we shall say that a structure envelopes a type-definable group $G$ to say that $G$ is the conjunction of definable groups.

Note that being enveloped is strictly stronger that being loosely enveloped. A stable structure is known to envelope type-definable groups and fields of finite arities [2, Hrushovski]. Consequently, in an omega-stable structure, a type-definable group of finite arity is definable, as is a type-definable field of finite arity in a superstable structure. Pillay and Poizat proved that a $\emptyset$-type-definable equivalence relation on a small structure is enveloped, provided that it be coarser than the equality of pure 1-types [9]. Kim generalised Pillay and Poizat's result to arbitrary $\emptyset$-type-definable equivalence relations on a small structure [4]. In [11], Wagner deduces from Kim's result that if a small structure loosely envelopes a $\emptyset$-type-definable group of finite

2000 Mathematics Subject Classification. 03C45, 03C60, 20L05, 20M99.

Key words and phrases. Small theory, simple theory, type-definable group, semi-group, field, ring, preorder, equivalence relation, category, groupoid.

The results of this paper form part of the author's doctoral dissertation, written in Lyon under the supervision of professor Frank O. Wagner. 
arity, it must envelope it. He asked whether a $\emptyset$-type-definable group in a small structure should be enveloped [11, Problem 6.1.14]. We shall show

Theorem. A Ø-type-definable category of finite arity in a small structure is the conjunction of definable categories.

As the notion of category both generalises preorders and semi-groups, the latter includes Kim's result and gives a positive answer to Wagner's question. It also gives a similar conclusion for a $\emptyset$-type-definable groupoid, which is a category where every morphism is invertible. As type-definable groupoids arise naturally in some structures (see [3, Hrushovski] and [1, Goodrick, Kolesnikov], this result might have an interest in itself. If we want to look at type-definable categories over an infinite set (but still of finite arity), we have to assume additional conditions, in our case that the ambiant theory be simple. Let us take for instance a $A$-type-definable group $G_{A}$ of finite arity. A compactness argument provides us with a definable set $X$ around $G_{A}$, on wich the group law is definable and associative. We obtain :

Theorem. Let $G_{A}$ be an A-type-definable group of finite arity in a small and simple structure.

i) If $G_{A}$ is included in some definable set $X$ such that boundedly many translates of $G_{A}$ cover $X$, then $G_{A}$ is the conjunction of definable groups.

ii) For any finite tuple $\bar{g}$ in $G_{A}$, there is a definable group containing $\bar{g}$.

For an $A$-type-definable (possibly skew) field $K_{A}$ of finite arity in a small and simple structure, the latter statement provides definable fields around every point, which give information about the structure of $K_{A}$ : it must be algebraically closed, and in positive characteristic, commutative.

\section{A FEW WOrds on StruCtures ENVEloping AlgEbraic STRUCTURES}

In the sequel, everything is inside some arbitrary universe $\mathfrak{M}$, who may have additional properties in the following sections.

Definition 1.1. A set $X$ is A-type-definable in $\mathfrak{M}$ if it is a subset of $\mathfrak{M}^{\alpha}$ for some ordinal $\alpha$, which is defined by a partial type with parameters in $A$. We call $\alpha$ the arity of $X$ in $\mathfrak{M}$.

An A-type-definable structure is any structure whose domain, functions and relations are $A$-type-definable. When considering type-definable groups in $\mathfrak{M}$ (and more generally, type-definable structures satisfying a given set of axioms $T$ ), we suppose that its type still defines a group (respectively still satisfies the axioms of $T$ ) in any elementary extension of $\mathfrak{M}$. In this section, every type-definable set considered will have finite arity in $\mathfrak{M}$. As we make no assumption on the ambiant structure, we may also assume in this section that every type-definable set considered is definable without parameters, by expanding the langage with possible parameters.

1.1. Equivalence relations, groups and fields. We begin by recalling a remark from $[8$, Poizat $]$.

Lemma 1.2. Let $X$ be a type-definable set of finite arity, and $\Gamma$ the graph of a type-definable map $f$ from $X$ to $X$. There are two definable sets $Y$ and $Z$ arround $X$ and a definable map $g$ from $Y$ to $Z$ so that $f$ be the trace of $g$ over $X$. 
Note however that $Z$ need not be a subset of $Y$ in general.

Proof. The type $\Gamma(x, y) \wedge \Gamma(x, z) \wedge y \neq z$ is inconsistent. The result follows by compactness.

We go on by stating simple, but new, observations. Next Proposition will be generalised to Proposition 1.14.

Proposition 1.3. If $\mathfrak{M}$ envelopes every equivalence relation, it also envelopes every group.

Proof. Let $G$ be a type-definable group in $\mathfrak{M}$. By Lemma 1.2, we may assume the group law to be definable. By compactness, there exists a definable set $X_{0}$ such that the group law be associative on $X_{0}$, and such that each element of $X_{0}$ have a unique inverse in $X_{0}$. Let $X_{1}, X_{2}, \ldots$ be a chain of definable subsets of $X_{0}$ whose intersection equals $G$. As $G$ is stable by multiplication, by compactness, there is some $X_{j}$, say $X_{1}$, such that $X_{1} \cdot X_{1}$ be a subset of $X_{0}$. We consider the equivalence relation $E$ on $X_{0}$ saying that $x$ and $y$ are related if $x y^{-1} \in G$. By hypothesis, $E$ is the conjunction of definable equivalence relations $E_{i}$. Note that an element $g$ in $X_{0}$ belongs to $G$ if and only if $1 E g$. By compactness, there exists some index $j$ such that $X_{1}$ contain $\left\{x \in X_{0}: x E_{j} 1\right\}$. Let us call this latter $J$. Then the set $G \cdot J$ is included in $J$. For instance, if $g \in G$ and if $y \in J$, then $g$ equals $g y y^{-1}$ and $g y$ belongs to $X_{0}$, so $g y E y$, hence $g y E_{j} 1$ by transitivity. Thus, if $H$ denotes the left stabiliser of $J$, that is, the set $\left\{g \in X_{0}: g J \subset J\right\}$, then the set $H \cap H^{-1}$ is a group in $X_{0}$ containing $G$. Note that if the ambient structure envelopes every equivalence relation, so does every $X_{i}$. It follows that every $X_{i}$ contains some definable group $H_{i}$ around $G$, and $G$ is the conjunction of every $H_{i}$.

Remark 1.4. Note that the converse fails, as there are superstable structures that do not envelope every equivalence relation [9, Exemple 2]. However, next section shows that envelopping preorders is equivalent to envelopping every semi-group.

Remark 1.5. Let $E$ be a type-definable equivalence relation, and let $E^{*}$ the typedefinable equivalence relation defined by :

$x E^{*} y$ if and only if there exists some $b$ such that $t p(b)=t p(x)$ and $b E z$

Recall from [9, Pillay Poizat] that $E$ is enveloped if and only if $E^{*}$, as well as every restriction of $E$ to a complete type are enveloped. Actually, replacing the equivalence relation $x y^{-1} \in G$ in the previous proof by $\exists b \models t p(x) \wedge b y^{-1} \in G$, one shows that the structure $\mathfrak{M}$ needs only envelope the equivalence relations coarser than the equality of types to envelope every group.

Proposition 1.6. If $\mathfrak{M}$ envelopes every group, it also envelopes commutative fields, and envelopes every (possibly skew) field with respect to integral rings.

Proof. Let $K$ be a type-definable field in this structure. By compactness, there is a definable set $X$ containing $K$ such that addition and multiplication be associative on $X$, and such that multiplication be distributive over addition. We may also assume that every element in $X$ have an additive and multiplicative inverse, and put $0^{-1}$ equal 0. Replacing $X$ by $X \cap-X \cap X^{-1} \cap-X^{-1}$, we may assume that $X$ equals $-X$ and $X^{-1}$. It follows that $X$ is integral. By hypothesis there exists a definable additive group $H$ inside $X$ and around $K^{+}$, and also a definable multiplicative 
group $M$ inside $H$ and around $K^{\times}$. Let $S$ be the set $\{h \in H: M \cdot h \subset H\}$. This is an additive subgroup of $H$ stabilised by left multiplication by $M$. Let $L$ be the set $\{h \in H: h \cdot S \subset S\}$. This is a definable integral ring containing $M$. If multiplication is commutative, for every $a, b, c, d$ in $L$, one has the equality

$$
a b^{-1}+c d^{-1}=(b d)^{-1}(a d+c b)
$$

so the product $L \cdot L^{-1}$ is a field containing $K$.

1.2. Preorders, semi-groups, rings and categories. We call a preorder any binary relation which is reflexive and transitive. A semi-group is any set with an associative binary operation. A semi-group might have no identity element.

Proposition 1.7. If $\mathfrak{M}$ envelopes every preorder, it also envelopes every semigroup.

Proof. Let $M$ be a type-definable semi-group. We may add a new constant 1 to $M$, and the set $\{(1, x, x),(x, 1, x): x \in M \cup\{1\}\}$ to the graph of multiplication, and assume that $M$ has an identity. Let $X_{0}$ be a definable set containing $M$ where the law is associative. Let $X_{1}$ be a definable set containing $M$ such that $X_{1} \cdot X_{1} \subset X_{0}$. We consider the preorder $R$ on $X_{0}$ defined by $x R y$ if and only if $x \in M y$, and finish as in the group case.

We shall show in the sequel that the converse is also true (see Propositions 1.10 and 1.11). As in the field case, and with a similar proof, we have

Proposition 1.8. If $\mathfrak{M}$ envelopes every semi-group, it also envelopes every (possibly non-commutative) ring.

Definition 1.9. A category is a two-sorted structure, the objects $O$, and morphisms $M$, together with applications $i_{0}$ and $i_{1}$ from $M$ to $O$ (saying that the morphism $m$ from $M$ goes from $i_{0}(m)$ to $i_{1}(m)$ ), a partial associative composition map $\circ$ from $M \times_{i_{0}, i_{1}} M$ to $M$ ( $m \circ n$ is defined when $i_{0}(m)$ equals $\left.i_{1}(n)\right)$, and an identity map $I d$ from $O$ to $M$ (such that $I d(x)$ be the identity morphism from $x$ to $x$ ).

On the sorts of objects of a given category, one can define a preorder by setting $a \leq b$ if there is a morphism from $a$ to $b$, as well as semi-groups $M_{a}$ whose elements are morphisms from $a$ to $a$ for any object $a$. Conversely, on the one hand, a preorder $\leq$ is a category with trivial semi-groups, and with one morphism for every couple $a, b$ satisfying $a \leq b$. On the other hand, a semi-group is a category with one single object and morphisms given by right multiplication by any element. Hence, the notion of category generalises both preorders and semi-groups.

Proposition 1.10. If $\mathfrak{M}$ envelopes any semi-group, it also envelopes any category.

Proof. Let $C$ be a type-definable category, with objects $O$ and morphisms $M$. The set $M$ has a partial structure of semi-group with law $\circ$, which can be extended to the whole of $M$ : let $o$ be a new object and 0 a new morphism from $o$ to $o$. Let $\bar{O}$ equal $O \cup\{o\}$, and $\bar{M}$ equal $M \cup\{0\}$. We extend $i_{0}, i_{1}$ and $\circ$ respectively to $\bar{i}_{0}, \bar{i}_{1}$ and $\bar{o}$ by setting $\bar{i}_{0}(0)=\bar{i}_{1}(0)=o$ and $0 \bar{\sigma} 0=0 \bar{\sigma} m=m \bar{\circ} 0=m \bar{\circ} n=0$ for all morphisms $m, n$ such that $i_{0}(m) \neq i_{1}(n)$; the law $\bar{o}$ is still type-definable (as $O$ has finite arity), and associative over $\bar{M}$. By hypothesis, $\bar{M}$ is the conjunction of definable semi-groups $\bar{M}_{i}$. By compactness, $i_{0}$ and $i_{1}$ are defined on $\bar{M}_{i}$ for 
sufficiently large $i$. Let $M_{i}$ equal $\bar{M}_{i}$ minus 0 and let $O_{i}$ equal $i_{0}\left(M_{i}\right) \cup i_{1}\left(M_{i}\right)$. $\left(O_{i}, M_{i}\right)$ is not a category yet as the map $I d$ need not be defined on $O_{i}$. But the equalities $I d\left(i_{1}(m)\right) \circ m=m$ and $n \circ \operatorname{Id}\left(i_{0}(n)\right)=n$ hold for all $m, n$ in $M$. By compactness, they must still hold for every $m, n$ in $M_{i}$ for some sufficiently large $i$. In particular, $I d$ is defined on $O_{i}$. One still need not have $\operatorname{Id}\left(O_{i}\right) \subset M_{i}$, but if it does not hold, by enforcing additional $I d$ maps for $O_{i} \backslash I d^{-1}\left(M_{i}\right)$ to $M_{i}$ and obvious composition laws, one can assume that $\left(O_{i}, M_{i}\right)$ is a category.

Proposition 1.11. If $\mathfrak{M}$ envelopes any category, it also envelopes any preorder.

Proof. A preorder $\leq$ on some set $X$ is a category $C$ with objects $X$, morphisms $\{(x, y): x \leq y\}$, and maps $i_{0}, i_{1}$, $\circ$ and $I d$ defined by $i_{0}(x, y)=x, i_{1}(x, y)=y$, $(x, y) \circ(y, z)=(x, z)$, and $\operatorname{Id}(x)=(x, x)$. By hypothesis, if $\leq$ is type-definable, it is the conjunction of definable categories $C_{i}$. By compactness, for sufficiently large $i$, the category $C_{i}$ is a preorder, i.e. there is at most one morphism between every ordered pair of objects.

Definition 1.12. A groupoid is a category whose morphisms are invertible.

Note that this generalises both the notions of groups and equivalence relations.

Remark 1.13. Similarly to the proof of Proposition 1.11, a structure which envelopes any groupoid also envelopes any equivalence relation.

Proposition 1.14. $\mathfrak{M}$ envelopes any equivalence relation if and only if it envelopes any groupoid.

Proof. We adapt the proof from the group case. Let $G$ be a groupoid, and let $O$ and $M$ be its objects and morphisms. By compactness, there are definable sets $X_{O}$ and $X_{M}$ containing $O$ and $M$, such that $i_{0}$ and $i_{1}$ be defined over $X_{M}$, and such that $I d$ be defined over $X_{O}$, and $\circ$ associative and defined over $X_{M}$, with in addition the equality $I d\left(i_{1}(m)\right) \circ m=m \circ I d\left(i_{0}(m)\right)=m$ holding for every $m$ in $X_{M}$. We may assume that $X_{M}$ equal $X_{M}^{-1}$. By compactness, there is some definable $Z_{M}$ containing $M$ with $Z_{M} \circ Z_{M}$ included in $X_{M}$. Let $E$ be the equivalence relation over $X_{M}$ defined by

$$
x E y \Longleftrightarrow i_{0}(x)=i_{0}(y) \wedge x \circ y^{-1} \in M
$$

By hypothesis, $E$ is the conjunction of definable equivalence relations $E_{i}$. Any element $x$ of $X_{M}$ belongs to $M$ if and only if $x E \operatorname{Id}\left(i_{0}(x)\right)$; by compactness, there is some index $j$ such that the inclusion $\left\{x \in X_{M}: x E_{j} I d\left(i_{0}(x)\right)\right\} \subset Z_{M}$ holds. Let $J$ equal $\left\{x \in Z_{M}: x E_{j} I d\left(i_{0}(x)\right)\right\}$ : it is stabilised by left multiplication by $M$. Namely, if $g$ is in $M$ and $y$ in $J$, and if $i_{0}(g)$ equals $i_{1}(y)$ then

$$
g=g \circ \operatorname{Id}\left(i_{0}(g)\right)=g \circ y \circ y^{-1}
$$

so $g \circ y$ is in $X$ hence $g \circ y E y$, thus $g \circ y E_{j} I d\left(i_{0}(y)\right)$. Let $H$ be the set $\{x \in J$ : $x \circ J \subset J\}$. $H$ is closed under composition. $\left(X_{O}, H \cap H^{-1} \cup \operatorname{Id}\left(X_{O}\right)\right)$ is a groupoid containing $G$. 


\section{Type-Definable StRuCtures in SMALL MOdels}

Definition 1. A structure is small if it has countably many $n$-types without parameters for every integer $n$.

In this section, we assume the ambiant structure $\mathfrak{M}$ to be small. We recall a theorem of Kim, using a result of Pillay and Poizat :

Fact 2.1. (Kim-Pillay-Poizat $[9,4])$ A small structure $\mathfrak{M}$ envelopes every $\emptyset$-typedefinable equivalence relation over $\mathfrak{M}$.

Note that [6, Krupiński, Newelski] gives an analytic proof of the previous theorem.

Remark 2.2. As $\mathfrak{M}$ is small, every finite cartesian power of $\mathfrak{M}$ is again small. The result fails for a type-definable equivalence relation over some infinite cartesian power of $\mathfrak{M}$, even in a $\aleph_{0}$-categorical structure : if $\mathfrak{M}$ is a dense linear order without end points, take the relation $E$ over $\mathfrak{M}^{\mathbf{Q}}$ saying that $x E y$ if and only if $x_{i}<y_{j}$ and $y_{i}<x_{j}$ for every $i<j$.

According to our previous observations, this answers Wagner's problem 6.1.14 in [11], and shows that a small structure envelopes any $\emptyset$-type-definable group, field and groupoid of finite arity. Recall that a definable small commutative field is either finite or algebraically closed [10, Wagner], and that a small division ring of positive characteristic a commutative field [7, Milliet].

Corollary 2.3. In a small structure, a $\emptyset$-type-definable commutative field of finite arity is finite or algebraically closed, and in positive characteristic, commutativity need not be assumed but follows.

Three main questions arise : what happens for $\emptyset$-type-definable groups of infinite arity? For $\emptyset$-type-definable semi-groups (even of finite arity)? And for $A$-typedefinable groups, where the set of parameters $A$ is allowed to be infinite? We tackle the two first questions in the next subsections, and give a partial answer to the third question in section 3 .

2.1. Preorders and semi-groups of finite arity. The following proposition is inspired from [9, Pillay, Poizat] and [4, Kim].

Proposition 2.4. A closed preorder on a denumerable Hausdorff compact space is the conjunction of clopen preorders.

Proof. Let $X$ be this Hausdorff compact space, and $R$ a closed preorder over $X$. Let $S^{c}$ stand for the complement of any subset $S$ of $X$. The space $X$ has a clopen basis, and $R$ is a closed set of tuples in $X \times X$. If $(x, y)$ is not in $R$, there exists a basic open set $O_{1} \times O_{2}$ outside $R$ containing the tuple $(x, y)$; the set $O_{1} \cap O_{2}$ is empty as $R$ is reflexive. We choose $O_{1}$ and $O_{2}$ such that $\left(O_{1} \cup O_{2}\right)^{c}$ have minimal CantorBendixson rank and degree, and write $Y$ for $\left(O_{1} \cup O_{2}\right)^{c}$. We show that $Y$ is empty ; otherwise, let $y$ be in $Y$ with maximal rank. If $\left(O_{1} \times\{y\}\right) \cap R$ and $\left(\{y\} \times O_{2}\right) \cap R$ are both non-empty, as $R$ is transitive, $\left(O_{1} \times O_{2}\right) \cap R$ is also non-empty, a contradiction. We may assume $\left(O_{1} \times\{y\}\right) \cap R$ to be empty. The set $O_{1} \times\{y\}$ is contained in the open set $R^{c}$. So we can choose a basic open set $Q_{2}$ containing $y$ with $O_{1} \times Q_{2} \subset R$. But $O_{1} \times\left(Q_{2} \cup O_{2}\right)$ is outside $R$. So $\left(O_{1} \cup O_{2} \cup Q_{2}\right)^{c}$ equals $Y^{c} \cap Q_{2}^{c}$, which misses 
$y$, a contradiction with the degree of $Y$ being minimal. So $Y$ is empty, $X$ equals $O_{1} \cup O_{2}$, and $O_{1} \times O_{1}^{c} \subset R^{c}$. Therefore, $R \subset\left(X \times O_{1}\right) \cup\left(O_{1}^{c} \times X\right)$, and $(a, b) \in R$ implies $(a, b) \in R_{x, y}$ where $R_{x, y}$ is the preorder defined by

$$
(a, b) \in R_{x, y} \Longleftrightarrow\left(a \in O_{1} \Rightarrow b \in O_{1}\right)
$$

We have shown that $(a, b) \in R$ is equivalent to $\bigwedge_{(x, y) \in R^{c}}\left((a, b) \in R_{x, y}\right)$.

Corollary 2.5. A small structure envelopes any $\emptyset$-type-definable preorder of finite arity $n$ which is coarser than equality between $n$-types without parameters.

Proof. Such a preorder $\leq$ induces a closed preorder $\lesssim$ on the space of $n$-types, defined by

$$
t p(a) \lesssim t p(b) \Longleftrightarrow a \leq b
$$

By Proposition 2.4, the preorder $\lesssim$ is the conjunction of definable preorders.

Proposition 2.6. A small structure envelopes every $\emptyset$-type-definable semi-group of finite arity.

Proof. Let $M$ be this semi-group. As smallness is preserved by adding finitely many parameters to the langae, without loss of generality we may assume that $M$ have a unit, and add it to the language. There is a definable set $X$ containing $M$ such that the law be associative on $X$. Let $R$ be a preorder on $X$ defined by

$$
x R y \Longleftrightarrow \exists z \models t p(y)(x \in M z)
$$

Note that if $x$ and $y$ have the same type over $\emptyset$, then $x$ and $y$ are in relation by $R$. By Corollary 2.5, $R$ is the conjunction of definable preorders $R_{i}$. Note that $m \in M$ if and only if $m R 1$. By compactness, there is some $j$ such that $\left\{x \in X: x R_{j} 1\right\} \subset X$. Let $J$ be the set $\left\{x \in X: x R_{j} 1\right\}$. It is left stabilised by $M:$ if $m$ is in $M$ and $y$ in $J$, then $m y \in M y$, so $m y R y$, thus $m y R_{j} 1$. Consider the left stabiliser of $J$ in $X$ : it is a semi-group containing $M$.

Remark 2.7. By compactness, a type-definable semi-group $M_{A}$ is the conjunction of type-definable semi-groups $M_{A_{i}}^{i}$ defined by countable types. It follows that every set $A_{i}$ is countable. By Proposition 2.6, an $\omega$-stable structure envelopes any $A$ type-definable semi-group were $A$ is arbitrary.

From Propositions 1.10 and 1.11, it follows :

Corollary 2.8. A small structure envelopes any $\emptyset$-type-definable preorder of finite arity.

2.2. Semi-groups of arbitrary arity. A semi-group $G$ with identity $1_{G}$ is said to almost act on a set $X$ if there is a map $G \times X \rightarrow X$. It acts on $X$ if in addition, for all $(g, h, x)$ in $G \times G \times X$, the equalities $(g h) \cdot x=g \cdot(h \cdot x)$ and $1_{G} \cdot x=x$ hold.

Lemma 2.9. In the small structure $\mathfrak{M}$, let $p$ be a partial type of finite arity, and let $X$ be the set $\left\{x \in \mathfrak{M}^{\omega}: \models p(x)\right\}$. Let $G$ be a semi-group acting on $X$ so that the action be $\emptyset$-type-definable in $\mathfrak{M}$. Then, there are formulae $f_{i}$, such that $X$ be the intersection of sets of the form $\left\{x \in \mathfrak{M}^{\omega}:=f_{i}(x)\right\}$ on which $G$ almost acts (with the same map). 
Proof. Let $f_{0}$ be any formula in $p$, and let $X_{0}$ be the set $\left\{x \in \mathfrak{M}^{\omega}:=f_{0}(x)\right\}$. By compactness, there is some formula $f_{1}$ in $p$ such that $G \cdot X_{1} \subset X_{0}$, where $X_{1}$ is the set $\left\{x \in \mathfrak{M}^{\omega}:=f_{1}(x)\right\}$. Let $X_{2}, X_{3} \ldots$ be a sequence of definable subsets of $X_{1}$ whose conjunction is $X$. Let $E$ be the equivalence relation on $X_{0}$ defined by

$$
x E y \Longleftrightarrow \exists g \in G(g \cdot x=y)
$$

$E$ is the conjunction of definable equivalence relations $E_{i}$. Note that $x \in X$ if and only if there exists some $a \in X$ with $a E x$. So there is some index $j$ such that $\{x \in$ $\left.\mathfrak{M}^{\omega}: \exists a \in X_{j}, a E_{j} x\right\} \subset X_{1}$. We show that $G$ acts on $\left\{x \in \mathfrak{M}^{\omega}: \exists a \in X_{j}, a E_{j} x\right\}$. We call $Y$ the latter set, and take some $g$ in $G$ and $x$ in $Y$; the product $g \cdot x$ is in $X_{0}$ so $x E g \cdot x$, hence $x E_{j} g \cdot x$ and $a E_{j} g \cdot x$.

Remark 2.10. The point of the previous lemma is that the semigroup $G$ may have infinite arity.

Remark 2.11. The result holds if the set $X$ is $A$-type-definable (of finite arity), as $E$ only involves parameters defining the semigroup $G$. Hence, if $G_{A}$ is an $A$-typedefinable group of finite arity, with a $\emptyset$-type-definable subgroup $H$, there exists a definable set $X$ containing $G_{A}$ stable under multiplication by $H$.

Proposition 2.12. In a small structure, a type-definable group is the intersection of definable sets each one equiped with a type-definable binary operation whose conjunction of graphs gives the group law.

Proof. Let $G$ be this group. As $G$ is the intersection of type-definable groups defined by countable types, we may assume that $G \subset \mathfrak{M}^{\omega}$ and that $G$ is the conjunction of countably many sets of the form $X_{i}=\left\{x \in \mathfrak{M}^{\omega}:=f_{i}(x)\right\}$ where $f_{i}$ are formulae. By compactness, we may assume that $G \cdot X_{1} \subset X_{0}$. For every integer $n$, let $E_{n}$ be the equivalence relation "to have the same $n$ first coordinates". On $X_{0}$, we set

$$
x R_{n} y \Longleftrightarrow \exists g, h \in G\left(g \cdot x E_{n} h \cdot y\right)
$$

Note that $x \in G$ if and only if $x R_{n} 1$ for all $n$. By compactness, there is an integer $n$ such that $R_{n} 1 \subset X_{1}$. Then, $R_{n} 1$ is stabilised left multiplicatively by $G$. As the type defining $R_{n} 1$ constrains only finitely many variables, by Lemma 2.9 , we may assume that $G$ almost acts on every $X_{i}$. By compactness, $X_{i}$ is stable under multiplication for sufficiently large $i$.

\section{TyPe-DEFINABLE GROUPS AND FIELDS IN A SMALL AND SIMPLE STRUCTURE}

3.1. Groups. In [4], Kim shows that the notion of strong type and Lascar strong type coincide in a small and simple theory, a necessary condition to eliminate hyperimaginaries. He proceeds in two steps, considering in the first one equivalence relations with boundedly many classes. We give an analogue of the first step for type-definable groups of finite arity.

In this last section, all type-definable groups and field considered will have finite arity.

Let $\mathfrak{M}$ be a $\kappa$-satured model of some theory $T$. For a set, bounded will mean strictly smaller than $\kappa$. An hyperimaginary is a class $a / E$ of some $a$ in $\mathfrak{M}^{\alpha}$ modulo a type-definable equivalence relation $E$ on $\mathfrak{M}^{\alpha}$, where $\alpha$ is a bounded ordinal. We write $A u t(\mathfrak{M} / A)$ for the group of automorphisms of $\mathfrak{M}$ fixing $A$ setwise. The action 
of $A u t(\mathfrak{M} / A)$ over $\mathfrak{M}$ naturally extends to hyperimaginaries. The bounded closure of some set $A$, written $b d d(A)$, is the set of hyperimaginaries whose orbit under $\operatorname{Aut}(\mathfrak{M} / A)$ is bounded. We will not define here what a simple theory is, but refer the reader to [11, Wagner] for more details. If $T$ is simple, two elements $a$ and $b$ have the same Lascar strong type over $A$, which we write "Lstp $(a / A)=L \operatorname{stp}(b / A)$ ", if and only if they have the same type over $b d d(A)$ (see [11, Lemma 3.2.13]). Let us recall the independence Theorem for Lascar strong types in simple theories.

Fact 3.1. (Kim-Pillay [5]) In a simple theory, let $A, B, C, b$ and $c$ satisfy

1) $A \subset B, A \subset C$ and $B \downarrow_{A} C$,

2) Neither $\operatorname{tp}(b / B)$, nor $t p(c / C)$ fork over $A$,

3) $\operatorname{Lstp}(b / A)=\operatorname{Lstp}(c / A)$.

Then there exists some a such that $t p(a / B C)$ extends both $t p(b / B)$ and $t p(c / C)$, such that $\operatorname{tp}(a / B C)$ does not fork over $A$, and such that $a, b$ and $c$ have the same Lascar strong type over A.

Two subgroups $G$ and $H$ of some group $F$ are commensurable if the indices $[G: G \cap H]$ and $[H: G \cap H]$ are bounded. The A-connected component of a group $G$ is the smallest $A$-type-definable group of bounded index in $G$. Every type-definable group in a simple theory has an $A$-connected component (see [11, Lemma 4.1.11]), which we will denote by $G_{A}^{0}$. When it exists, $G_{A}^{0}$ is always a normal subgroup of G.

Lemma 3.2. Let $X$ be a definable set in any structure with a definable composition law (the product of two elements of $X$ may be outside $X$ ) such that the product of every six elements of $X$ be defined and associative. Let $G_{A}$ be an A-type-definable group inside $X$ sharing the same composition law as $X$. If the A-connected component of $G_{A}$ is contained in some definable group $H$ (with same law) in $X$, then $G_{A}$ is contained in a definable group included in $H \cdot G_{A}$.

Proof. As the product of five elements of $X$ is well defined, the group $\bigcap_{h \in H \cdot G_{A}} H^{h}$ is well-defined definable. Let us call it $N$. Note that

$$
N=\bigcap_{h \in H \cdot G_{A}} H^{h}=\bigcap_{g \in G_{A}} H^{g}=\bigcap_{\bar{g} \in G_{A} / G_{A} \cap G} H^{h}
$$

As $G_{A} \cap H$ has finite index in $G_{A}$, it follows that $N$ is definable. Moreover, it is a subgroup invariant under conjugation by elements of $G_{A}$. As $N$ is the intersection of conjugates of $H$ under $G_{A}$, and as the connected component $G_{A}^{0}$ is normal in $G_{A}$, the group $N$ contains $G_{A}^{0}$. The product $N \cdot G_{A}$ is a definable group containing $G_{A}$.

A family $\mathfrak{H}$ of type-definable subsets of $\mathfrak{M}^{\alpha}$ is uniformly type-definable if there are two partial types $p(x, y)$ and $q(z)$ such that

$$
\mathfrak{H}=\left\{\left\{x \in \mathfrak{M}^{\alpha}:=p(x, a)\right\}:=q(a)\right\}
$$

If $q$ and $p$ are types over $A$, the family $\mathfrak{H}$ is uniformly A-type-definable.

Recall that in a type-definable group $G$ with simple theory, an element $g$ is generic over $A$ if for every $h \downarrow_{A} g$ in $G$, we have $h g \downarrow A, h$. Recall [11, Lemma 4.1.19] and [11, Remark 4.1.20], which together give : 
Fact 3.3. (Wagner [11]) In a simple structure, let $G$ be a definable set with a definable composition law having an identity $1_{G}$, and such that the product of every three elements of $G$ be defined and associative, and such that any element have a right and left inverse in $G$. In $G$, let $X$ be an A-type-definable set containing $1_{G}$, such that for all $x$ and $y$ in $X$ independent mover $A$, the product $x^{-1} y$ be in $X$. Then $X \cdot X$ is a type-definable group and $X$ is generic in $X \cdot X$. Actually, $X$ contains every generic type of $X \cdot X$.

Note that there indeed needs an extra assumption that $1_{G} \in X$ which is missed in Wagner's book.

Let us now point out a result from Wagner's proof of [11, Theorem 4.5.13] :

Fact 3.4. In a simple structure, let $X$ be an A-type-definable set with an A-definable composition law (the product of two elements of $X$ may be outside X). Let $\mathfrak{H}$ be a uniformly A-type-definable family of pairwise commensurable groups in $X$ sharing the same composition law as $X$. If $X$ contains $\mathfrak{H} \cdot \mathfrak{H} \cdot \mathfrak{H} \cdot \mathfrak{H}$, there exists an A-typedefinable group $N$ (with same law) inside $\mathfrak{H} \cdot \mathfrak{H} \cdot \mathfrak{H} \cdot \mathfrak{H}$ which is commensurable with every $H$ in $\mathfrak{H}$.

Proof. Suppose that $\mathfrak{H}$ be given as $\{H(a): \models \pi(a)\}$ where $\pi$ is a partial type over A. Put

$$
N_{A}=\{x \in X:(\exists y \models \pi)(y \underset{A}{\downarrow} x \wedge x \in H(y))\}
$$

$N_{A}$ is a $A$-type definable set (i.e. a type-definable set wich is invariant under $A$-automorphisms)

$\mathrm{A}=\mathrm{bdd} \mathrm{A}=$ Gamma?

Pi complet ??

Let $x$ and $y$ be two elements of $N_{A}$ such that $x \downarrow_{\Gamma} y$. We show that $x^{-1} y$ is inside $N_{\Gamma}$ : there are elements $a$ and $b$ realising $p$ such that $a \downarrow_{\Gamma} x, b \downarrow_{\Gamma} y$, and such that $x$ belong to $H_{a}$ and $y$ to $H_{b}$. According to the Independence Theorem 3.1, there exists some $c$ realising $t p(a / x \Gamma) \cup t p(b / y \Gamma)$ such that $c \downarrow_{\Gamma} x, y$. Thus $c \downarrow_{\Gamma} x^{-1} y$. But $x$ and $y$ are in $H_{c}$ so $x^{-1} y$ is in $H_{c}$ too ; a fortiori, $x^{-1} y$ is in $Y$. After Fact 3.3 , the product $N_{\Gamma} \cdot N_{\Gamma}$ is a $\Gamma$-type-definable group. Let us consider the group

$$
\bigcap_{\sigma \in \operatorname{Aut}(\mathfrak{C} / \bar{g})} \sigma\left(N_{\Gamma} \cdot N_{\Gamma}\right)
$$

Recall that $\Gamma=b d d(\bar{g}, \bar{h})$, hence this is a bounded, $\bar{g} \bar{h}$-type-definable intersection containing $\operatorname{acl}(\bar{g}) \cap G_{A}$.

Proposition 3.5. In a small and simple structure, let $Z$ be a definable set, and $G_{A}$ be an A-type-definable group inside $Z$, such that boundedly many translates of $G_{A}$ cover $Z$. Then $Z$ envelopes $G_{A}$.

Proof. We may restrict $Z$ and suppose that the group law be defined and associative on $Z$. By compactness there is some definable set $Y$ containing $G_{A}$ such that $Y \cdot Y \cdot Y \cdot Y \subset Z$. By suppressing a finite parameter, we may suppose that $Z, Y$, 
$1_{G_{A}}$ and the group law are $\emptyset$-definable. Let $\mathfrak{H}$ be the set $\left\{G_{B}: B \models t p(A / \emptyset)\right\}$. The elements in $\mathfrak{H}$ are pairwise commensurable. According to Fact 3.4, there exists a $\emptyset$-type-definable group $N \subset Z$ which is commensurable with $G_{A}$; hence, $N$ is contained in a definable group $M \subset Z$ by Proposition 1.3 and Fact 2.1. But $N$ contains the connected component of $G_{A}$, so $G_{A}$ is contained in some definable group included in $M \cdot G_{A}$ according to Lemma 3.2.

Without the assumption that boundedly many translates have to cover the group $G_{A}$, the author could not succeed to get the conclusion of Proposition 3.5. But at least can he state local results :

Lemma 3.6. In a simple structure, let $G_{A}$ be an A-type-definable group, and let $\bar{g}$ be a finite tuple of elements in $G_{A}$. There is a finite set $B$ and a B-type-definable group containing $\operatorname{acl}(\bar{g}) \cap G_{A}$.

Proof. The group law is defined and associative on a definable set $X$ containing $G_{A}$. By compactness, there is a $h$-definable set $Y$ inside $X$ such that $Y \cdot Y \subset X$. Let $\Gamma$ be the bounded closure of $\bar{g} \cup \bar{h}$. Let $N_{\Gamma}$ be the set

$$
\left\{x \in Y: \exists A^{\prime} \models \operatorname{tp}(A / \Gamma)\left(A^{\prime} \underset{\Gamma}{\downarrow} x \wedge x \in G_{A^{\prime}}\right)\right\}
$$

$N_{\Gamma}$ is a $\Gamma$-type-definable set containing $\operatorname{acl}(\bar{g}) \cap G_{A}$. Let $x$ and $y$ be two elements of $N_{\Gamma}$ such that $x \downarrow_{\Gamma} y$. We show that $x^{-1} y$ is inside $N_{\Gamma}$ : there are elements $A^{\prime}$ and $A^{\prime \prime}$ realising $t p(A / \Gamma)$ such that $A^{\prime} \downarrow_{\Gamma} x, A^{\prime \prime} \downarrow_{\Gamma} y$, and such that $x$ belong to $G_{A^{\prime}}$ and $y$ to $G_{A^{\prime \prime}}$. According to the Independence Theorem 3.1, there exists some $A^{\prime \prime \prime}$ realising $\operatorname{tp}\left(A^{\prime} / x \Gamma\right) \cup \operatorname{tp}\left(A^{\prime \prime} / y \Gamma\right)$ such that $A^{\prime \prime \prime} \downarrow_{\Gamma} x, y$. Thus $A^{\prime \prime \prime} \downarrow_{\Gamma} x^{-1} y$. But $x$ and $y$ are in $G_{A^{\prime \prime \prime}}$ so $x^{-1} y$ is in $G_{A^{\prime \prime \prime}}$ too ; a fortiori, $x^{-1} y$ is in $Y$. After Fact 3.3, the product $N_{\Gamma} \cdot N_{\Gamma}$ is a $\Gamma$-type-definable group. Let us consider the group

$$
\bigcap_{\sigma \in \operatorname{Aut}(\mathfrak{C} / \bar{g})} \sigma\left(N_{\Gamma} \cdot N_{\Gamma}\right)
$$

Recall that $\Gamma=b d d(\bar{g}, \bar{h})$, hence this is a bounded, $\bar{g} \bar{h}$-type-definable intersection containing $\operatorname{acl}(\bar{g}) \cap G_{A}$.

Corollary 3.7. In a small and simple structure, let $\bar{g}$ be a finite tuple of an $A$ type-definable group $G_{A}$. There is a definable group containing acl $(\bar{g}) \cap G_{A}$.

\subsection{Fields.}

Lemma 3.8. In a simple structure, let $K$ be a definable set with two definable composition laws (the sum and product of two elements of $K$ may be outside $K$ ), each having an identity 0 and 1 respectively (which are a constant in the language), and such that the sum and product of every three elements of $G$ be defined and associative. We assume that multiplication is distributive over addition and that any element in $K$ has a right and left inverse in $K$ for each law. In $K$, let $X$ be an A-type-definable set containing 0 and 1 , and such that for all $x$ and $y$ in $X$ independent over $A$, the sum $x-y$ and product $x^{-1} y$ be in $X$. Then $X+X$ is a type-definable field. 
Proof. Note first that $X$ equals $-X$ and $X^{-1}$ as $X$ contains 0 and 1 . According to Fact $3.3, X+X$ is an additive group ; we need just show that $X \cdot X$ is included in $X+X$, for we shall have

$$
(X+X) \cdot(X+X) \subset X \cdot X+X \cdot X+X \cdot X+X \cdot X \subset X+X
$$

Let $p$ be an additive generic type in of $X+X$. Then $p$ is in $X$. Let $g$ and $g^{\prime}$ be in $X$, and let $h$ be in $p$ such that $h \downarrow g, g^{\prime}$. Then, $h \downarrow_{g^{\prime}} g$ and $h+g^{\prime} \downarrow_{g^{\prime}} g$. Moreover, we have $g^{\prime}+h \downarrow g^{\prime}$, so $g^{\prime}+h \downarrow g$ by transitivity. Hence, $g g^{\prime}+g h$ is in $X$. As $h^{-1}$ is in $X$ and $g \downarrow h^{-1}$, the product $g h$ belongs to $X$ and $g g^{\prime}$ to $X+X$.

Lemma 3.9. In a simple structure, let $\bar{g}$ be finite tuple of an A-type-definable (possibly skew) field $K_{A}$. There is a finite set $B$ and a $B$-type-definable field containing $\operatorname{acl}(\bar{g}) \cap G_{A}$.

Proof. Let $X$ be a definable set where addition and multiplication are defined and associative, and where multiplication is distributive over addition, and let $Y$ be a $\bar{h}$-definable subset of $X$ such that $Y \cdot Y$ and $Y+Y$ are in $X$. Let $B$ be the bounded closure of $\bar{g} \cup \bar{h}$, and let $L_{B}$ be the set

$$
\left\{x \in Y: \exists C \models \operatorname{tp}(A / B)\left(C \underset{B}{\downarrow} x \wedge x \in K_{C}\right)\right\}
$$

$L_{B}$ is a $B$-type-definable set containing $\operatorname{acl}(\bar{g}) \cap G_{A}$. If $x$ and $y$ are two elements of $L_{B}$ independent over $B$, then $x^{-1} y$ and $x-y$ also lie in $L_{B}$. According to Fact 3.3 , the set $\bigcap_{\sigma \in A u t(\mathfrak{C} / \bar{g})} \sigma\left(L_{B}+L_{B}\right)$ has the required properties.

Corollary 3.10. In a small and simple structure, let $\bar{g}$ be a finite tuple of an Atype-definable (possibly skew) field $K_{A}$. Then there is a definable field containing $\operatorname{acl}(\bar{g}) \cap K_{A}$.

Corollary 3.11. In a small and simple structure, an A-type-definable commutative field of finite arity is finite or algebraically closed, and in positive characteristic, commutativity need not be assumed but follows.

Proof. Let $K_{A}$ be commutative field in a small and simple structure. If it is infinite, by compactness, there is an element $x$ of infinite order in $K_{A}$. Let $P$ be a polynomial with coefficients in $K_{A}$. According to Corollary 3.10, for every definable set $X$ containing $K_{A}$, there is a definable field $L_{X}$ in $X$ which contains $x$ and the coefficients of $P$. By [10, Wagner], $L_{X}$ is algebraically closed. The field $\bigcap_{X \supset K_{A}} L_{X}$ is an algebraically closed subfield of $K_{A}$ which contains every coefficient of $P$.

If $K_{A}$ has positive characteristic and is not assumed to be commutative, let $x$ and $y$ be in $K_{A}$. By Corollary 3.10, there is a definable field containing $x$ and $y$, so $x$ and $y$ commute after [7, Milliet].

\section{REFERENCES}

[1] John Goodrick and Alexei Kolesnikov, Groupoids, covers, and 3-uniqueness in stable theories, The Journal of Symbolic Logic 75, 3, 905-929, 2010.

[2] Ehud Hrushovski, Contributions to stable model theory, PhD, Berkeley, 1986.

[3] Ehud Hrushovski, Groupoids, imaginaries and internal covers, preprint, 2006.

[4] Byunghan Kim, A Note on Lascar Strong Types in Simple Theories, The Journal of Symbolic Logic 63, 3, 926-936, 1998. 
[5] Byunghan Kim and Anand Pillay, Simple theories, Annals of Pure and Applied Logic 88, 149-164, 1997.

[6] Krysztof Krupiński and Ludomir Newelski, On bounded type-definable equivalence relations, Notre Dame Journal of Formal Logic 43, 4, 231-242, 2002.

[7] Cédric Milliet, Small Skew fields, Mathematical Logic Quarterly 53, 86-90, 1, 2007.

[8] Bruno Poizat, Groupes Stables, Nur Al-Mantiq Wal-Ma'rifah, 1987.

[9] Anand Pillay and Bruno Poizat, Pas d'imaginaires dans l'infini, The Journal of Symbolic Logic 52, 2, 400-403, 1987.

[10] Frank O. Wagner, Small fields, The Journal of Symbolic Logic 63, 3, 995-1002, 1998.

[11] Frank O. Wagner, Simple Theories, Kluwer Academic Publishers, Dordrecht, NL, 2000.

Université de Lyon, Université Lyon 1

Institut Camille Jordan, UMR 5208 CNRS

43 BOULEVARD DU 11 NOVEMBRE 1918

69622 Villeurbanne Cedex, France

(Current address) Université Galatasaray

Faculté de Sciences et de Lettres

Çiragan Caddesi n ${ }^{\circ} 36$

34357 Ortaköy, Istamboul, Turquie

E-mail address: milliet@math.univ-lyon1.fr 